alcohol without feeling its effect. Participants also reported using marijuana throughout the day while driving, at work, or in class. Frequent patterns noted for the study included using two drugs at the same time or right before or after each other with alcohol used throughout the day. Participants also gave feedback on our Poly Substance Use (PSU) assessment that captures exact patterns so that the most common can be translated for the rodent models. DISCUSSION/SIGNIFICANCE OF IMPACT: Our focus group discussions provided detailed information on patterns, sequence, quantity, and types of poly substance use that could be useful for developing a poly substance use assessment in the collection of data for rodent models to understand effects of poly substance use.

4269

Frequent emergency department use among homeless individuals seen in emergent care: High risks of opioidrelated diagnoses and adverse health services utilization outcomes

Ayae Yamamoto ${ }^{1}$, Lillian Gelberg, MD, MSPH ${ }^{2}$, Yusuke Tsugawa, MD, $\mathrm{PhD}^{2}$, Gerald Kominski, $\mathrm{PhD}^{2}$, and Jack Needleman, $\mathrm{PhD}^{2}$

${ }^{1}$ David Geffen School of Medicine at UCLA; ${ }^{2}$ UCLA Fielding School of Public Health

OBJECTIVES/GOALS: Using multi-state discharge data, to identify predictors of frequent emergency department (ED) use among the homeless patients seen in emergent care, and to compare frequent versus less frequent homeless ED users for their risk of serious health services utilization outcomes. METHODS/STUDY POPULATION: Based on the State Emergency Department Database and the State Inpatient Database, homeless individuals $(\mathrm{n}=88,541)$ who made at least one ED visit in four states (Florida, Maryland, Massachusetts, and New York) in 2014. In this retrospective cross-sectional analysis, patient-level demographic and clinical factors were assessed as predictors for increased ED use. Risks of opioid overdose, opioid-related hospital admission/ED visit, in-hospital mortality, mechanical ventilation, and number of hospitalizations were compared between individuals with 4 or more vs. $2-3$ vs. 1 ED visit(s), adjusting for potential confounders including hospital fixed effects (allowing for within hospital comparisons). RESULTS/ANTICIPATED RESULTS: Higher rates of ED use were associated with Medicare coverage $<65$; primary diagnosis of alcohol abuse, asthma, or abdominal pain; and co-morbidity of alcohol abuse, psychoses, or chronic pulmonary disease. Individuals with $\geq 4$ visits had significantly higher adjusted risk of opioid overdose (3.7\% vs. $1.2 \%$ vs. $1.0 \%$ ), opioid-related hospitalizations/ED visits ( $17.9 \%$ vs. $8.5 \%$ vs. $6.6 \%)$, mechanical ventilation $(9.8 \%$ vs. $7.0 \%$ vs. $4.7 \%$ ), and greater \# of hospitalizations ( 3.2 vs. 1.3 vs. 0.8 ) compared to individuals with 2-3 or 1 ED visit. Individuals with $\geq 4$ and 2-3 ED visits had similar but increased risks of in-hospital mortality compared to individuals with $1 \mathrm{ED}$ visit (2.8\% vs. $2.8 \%$ vs. $2.3 \%)$. DISCUSSION/SIGNIFICANCE OF IMPACT: Homeless patients who were high ED users were more likely to be hospitalized and have other adverse outcomes. These findings encourage targeted interventions (i.e. housing) for the high-utilizer homeless population to reduce the burden of serious outcomes and costs for the patient and society.

\section{Health and HIV: Weight status associations with multiple} co-morbidities

Kierra Renee Butler ${ }^{1}$, Faye R. Harrell, MSN, CRNP, Jeffrey Robinson, $\mathrm{PhD}$, Bridgett Rahim-Williams, PhD, MA, MPH, and Wendy A.

Henderson, PhD, MSN, CRNP

${ }^{1}$ National Institutes of Health

OBJECTIVES/GOALS: Highly Active Antiretroviral Therapy (HAART) is beneficial for managing HIV infection, however the long-term use of HAART may be problematic for healthy weight maintenance. The aim of the study was to investigate the association of race, weight status, and co-morbidities among individuals with HIV. METHODS/STUDY POPULATION: Self-reported data from 283 participants who completed the Symptom Checklist, the Co-Morbidity Questionnaire, and the Sociodemographic Questionnaire were included in the data analyses. Data were analyzed using Latent Class Analysis on JMP 13. Approximately 50\% of participants self-identified as Black, $69 \%$ as male, and $35 \%$ as having AIDS. Participants' age ranged from 25 to 66 years (mean age $=43.70$ years, $\mathrm{SD}=8.14$ ). Participants were grouped by race (selfreported Black or non-Black), and then each group was clustered based on the top three most prevalent symptoms. The clusters identified were least symptomatic, weight gain, and weight loss. RESULTS/ANTICIPATED RESULTS: The non-Black weight gain cluster reported a higher incidence of AIDS (70.6\% vs 38.2\%), nausea ( $70.6 \%$ vs $17.6 \%)$, diarrhea ( $70.6 \%$ vs $26.5 \%)$, and shortness of breath ( $58.8 \%$ vs $20.6 \%$ ) compared to the Black weight gain cluster. The Black weight loss cluster reported a higher incidence of cardiovascular symptoms including chest palpitations (42.2\% vs $2.7 \%$ ), chest pain $(44.4 \%$ vs $8.1 \%)$, and shortness of breath $(73.3 \%$ vs $35.1 \%)$ and a higher incidence of all GI symptoms with the most prominent being diarrhea $(71.1 \%$ vs $48.6 \%)$ compared to the nonBlack weight loss cluster. DISCUSSION/SIGNIFICANCE OF IMPACT: Future studies supporting these results will assist practitioners to target treatments that may prevent adverse health outcomes for individuals with HIV on HAART. Further studies will also assist with setting standards that allow practitioners to provide personalized care for individuals with HIV on HAART.

4031

Heart Transplant Candidates Listed at Low First-Offer Organ Acceptance Rate Centers are More Likely to Die Waiting

Ashley Y Choi ${ }^{1}$, Michael S. Mulvihill ${ }^{2}$, Hui-Jie Lee ${ }^{3}$, Congwen Zhao ${ }^{3}$, Maragatha Kuchibhatla ${ }^{3}$, Jacob N. Schroder ${ }^{2}$, Chetan B. Patel ${ }^{2}$, Christopher B. Granger ${ }^{2}$, and Matthew G. Hartwig ${ }^{2}$

${ }^{1}$ Duke University; ${ }^{2}$ Duke University Medical Center; ${ }^{3}$ Department of Biostatistics, Duke University

OBJECTIVES/GOALS: We sought to examine: 1) variability in center acceptance patterns for heart allografts offered to the highest-priority candidates, 2) impact of this acceptance behavior on candidate survival, and 3) post-transplantation outcomes in candidates who accepted first rank offer vs. previously declined offer. METHODS/STUDY POPULATION: In this retrospective cohort 